\title{
Interplay of weather variables in triggering the transmission of SARS-CoV-2 infection in Asia
}

\author{
Arpita Dalal ${ }^{1} \cdot$ Piyush Pandey ${ }^{2}$
}

Received: 22 January 2021 / Revised: 13 March 2021 / Accepted: 19 April 2021 / Published online: 17 May 2021

(c) Society for Environmental Sustainability 2021

\begin{abstract}
Weather variables are one of the crucial factors affecting respiratory infectious diseases; however, the effect of weather variables on the coronavirus disease 2019 (COVID-19) is still inconclusive and varies in different regions. The present study investigated the effects of weather variables (maximum temperature, MT; relative humidity, RH; wind speed, WS; precipitation, PR; and dew point, DP) on daily infection and death cases in three lockdown phases in Asia as of November 1, 2020. Generalized additive lag model was used to analyze the risk associated with weather variables, with confounders like median age of the national population, population density, country and lockdown phases. Our findings revealed that during lockdown phases all five weather variables show association with daily confirmed, and death cases. On the other hand, PR (pre-lockdown phase) and DP (lockdown phase) showed positive association with both daily confirmed and death cases. Throughout the three lockdown phases MT, RH and PR showed strong positive associations with daily confimed/ death cases. A lag period of 0-4-days possess higher risk of infection and death due to the varied ratios of different weather variables. The relative risk indicated that the infection and mortality risk was higher in India as compared to the rest of the countries. Here, unique combination of weather variables together with higher population density makes this region as one of the hotspots for COVID-19. This shows that the COVID-19 pandemic may be suppressed or enhanced with combination of different weather conditions together with factors like population density and median age of the country, which shall be useful for better implementation of health policies and further preparedness in Asia.
\end{abstract}

Keywords COVID-19 $\cdot$ Relative risk $\cdot$ Mortality risk $\cdot$ Infection $\cdot$ Mortality $\cdot$ Weather variables

\section{Introduction}

Severe acute respiratory syndrome coronavirus 2 (SARS$\mathrm{CoV}-2$ ), a strain of coronavirus is responsible for coronavirus disease 2019 (COVID-19), a predominantly respiratory ailment which is responsible for the COVID-19 pandemic (Gorbalenya et al. 2020). COVID-19 was first reported in Wuhan, China in December 2019 which soon spread to different parts of the world despite many restrictions in travel and so many preventive measures taken. Therefore, it was declared a pandemic by World Health Organization (WHO) because of its rapid spread throughout the world ( $\mathrm{Li}$ et al.

Piyush Pandey

piyushddn@gmail.com; ppmicroaus@gmail.com

1 Department of Ecology and Environmental Science, Assam University, Silchar 788011, India

2 Department of Microbiology, Assam University, Silchar 788011, India
2020). Previous studies showed weather variables like temperature and relative humidity can significantly help in occurrence and transmission of many infectious diseases, including severe acute respiratory syndrome (SARS) (Djennad et al. 2018; Tan et al. 2005). Recently, it was observed that weather variables, particularly mean temperature and relative humidity have significant associations with the occurrence of COVID-19 reported from different regions of the world (Bashir et al. 2020; Lin et al. 2020; Liu et al. 2020; Prata et al. 2020; Wu et al. 2020; Xie and Zhu 2020). Few researchers also considered diurnal temperature, wind speed, precipitation and dew point and found significant association with COVID-19 cases (Ma et al. 2020; Menebo 2020; Sahin 2020).

It has been observed that the lethality in temperate countries is more as compared to tropical countries due to presence of few unique mutations in SARS-CoV-2 genes (Islam et al. 2020). Mutations in virulence genes of SARS-CoV-2 was observed in several countries of Europe, Asia and 
Middle-East, yet the loss of human lives due to COVID-19 has been relatively lower particularly in Asia and MiddleEast as compared to Europe (Islam et al. 2020; Maurya et al., 2020). Therefore, the significance of weather variables is important for the occurrence and spread of COVID-19 and its rate of lethality. Due to this pandemic, most of the countries of the world have imposed partial or complete social restrictions resulting in prohibition of mass transportation and industrial activities. As a result, the air quality and overall environmental health has improved with drastic reductions in pollution (Bera et al. 2020; Mahato et al. 2020).

Most of works have concentrated in either at one country or taking the entire world but no work has been done taking only Asian continent which is the hub of COVID-19 with maximum cases. Moreover, no work has been done to see the effect of weather variables on the COVID-19 daily confirmed and death cases taking three lockdown stages in consideration. We, therefore, aim to determine the role of selected meteorological factors with daily confirmed and death cases of COVID-19 in selected countries of Asian continent with focus on three stages of lockdown period. This study establishes that combinations of different environmental variables have significant role in the increase or decrease of COVID-19 related infections and death.

\section{Materials and methods}

\section{Data collection}

Daily confirmed/infection and death cases of COVID-19 data were collected from 27 countries of Asian continent, excluding west Asian countries and Russia (Table S1). Data were collected till November 1, 2020 which were divided into three lockdown phases (pre-lockdown, lockdown and post-lockdown). Different countries have announced lockdown on different dates. Both partial and full lockdowns have been considered from the initial date of lockdown imposed (Table S1).

Collection of daily confirmed and death cases were collected from Johns Hopkins University, whereas the daily meteorological data were collected from the power project of NASA (Table S2). Among the meteorological data, maximum temperature (MT, ${ }^{\circ} \mathrm{C}$ ), average relative humidity (RH, $\%$ ), average wind speed (WS, $\mathrm{m} \mathrm{s}^{-1}$ ), precipitation (PR, mm day $^{-1}$ ) and average dew point $\left(\mathrm{DP},{ }^{\circ} \mathrm{C}\right)$ were considered for the study.

Median age and population density data of the 27 countries were also collected as potential confounders (Table S2). Median age indicates the population aging of a country's population. The occurrence of higher number of COVID-19 cases are observed in population with upper levels of aging (Verity et al. 2020). On the other hand, population density is the number of people per square mile of land area. A densely populated country can trigger the spread of epidemics (Khairat et al. 2020).

\section{Statistical analyses}

Descriptive analyses were performed at three lockdown phases. Multiple regression analyses were performed to identify relation between weather variables and daily confirmed and death cases. Cluster analyses using Bray-Curtis distance was used to identify the dissimilarity among the lockdown phases based on daily confirmed and death cases. A generalized additive model (GAM) with quasipoisson family (residual overdispersed as compared to degrees of freedom) was used in the present study to analyze the association between the selected weather variables with daily confirmed and death cases, separately as used in the previous studies (Almeida et al. 2010; Ma et al. 2020). First, weather variables were transformed with cross-basis matrix where a lag of 10 days were generated (0-10). Second, we built the GAM model for association of daily confirmed and death outcomes with weather variables. Important confounding factors were also added to this model such as 'country' and 'lockdown phase' as a categorical variable, and 'median age' and 'population density' as a numerical variable. Matching with several recent time-series studies (Cheng and Kan 2012; Zeng et al. 2016), the penalized smoothing spline functions were incorporated to control the effects of confounding factors, such as time, median age and population density. The core GAM equation is:

$$
\begin{aligned}
\log \left(Y_{t}\right)= & \alpha+\beta X_{t}+\text { Country }+ \text { Lockdown phase } \\
& +s(\text { Median age })+s(\text { Population density }) \\
& +s(\text { time }, d f)
\end{aligned}
$$

where $t$ is the day of the observation; $\alpha$ is the intercept; $\beta$ is the regression coefficient; $X_{t}$ is the daily weather variables on day $t ; s()$ refers the smoother based on the penalized smoothing spline; time is the date of observation and $d f$ is degrees of freedom.

After establishing the core model, relative risk (RR) was calculated adjusting for various confounders. The RR is defined as "the ratio of the probability of occurring of confirmed/death cases at a certain value of a weather variable to the probability of the event occurring at a reference value of the same weather variable'. All the statistical analyses were conducted using $\mathrm{R}$ software (version 4.0.2) (R Core Team 2020) and GAM model using 'dlnm' package (version 2.4.2) in $\mathrm{R}$ (Gasparrini 2011). The effect estimates were expressed in RR with per one-unit increase in weather variables. 


\section{Results}

\section{Descriptive analysis and cluster analysis}

A total of 10,607,067 cumulative confirmed cases and 171,537 cumulative death cases were documented till November 1, 2020. Overall highest daily confirmed and death cases were observed in India during post-lockdown phase till November 1, 2020. On the other hand, Laos has the lowest daily confirmed cases recorded (Fig. 1). Overall,
MT ranged from -7.90 to $45.82^{\circ} \mathrm{C}$, and $\mathrm{RH}$ ranged from 3.82 to $100 \%$. Average wind speed ranged from 0 to $11.20 \mathrm{~m} \mathrm{~s}^{-1}$, and PR ranged from 0 to $217.81 \mathrm{~mm} \mathrm{day}^{-1}$. Average dew point ranged from -32.77 to $27.43{ }^{\circ} \mathrm{C}$ during the study period.

Based on Bray-Curtis distance of cluster analysis it has been observed that pre- and post-lockdown has the most similar rate of daily confirmed and death cases, whereas lockdown period was the most dissimilar lockdown phase for both daily confirmed and death cases (Fig. 2).

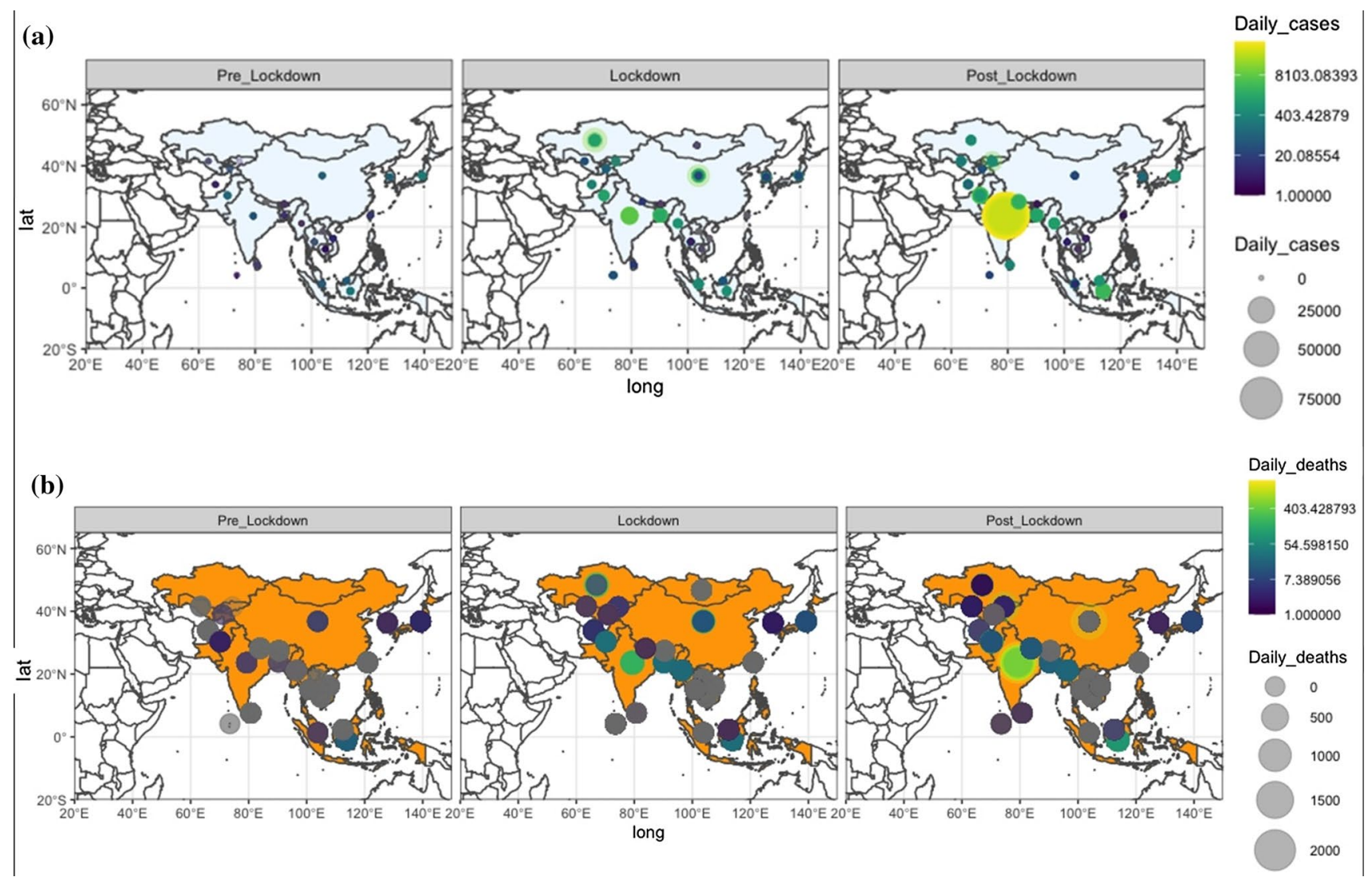

Fig. 1 Global distribution of $\mathbf{a}$ daily confirmed and $\mathbf{b}$ daily death cases of covid-19 cases till 1st November, 2020

Fig. 2 Cluster analysis using Dendrogram for Bray-Curtis distance of dissimilarity of three lockdown phase for a daily confirmed cases and $\mathbf{b}$ daily death cases

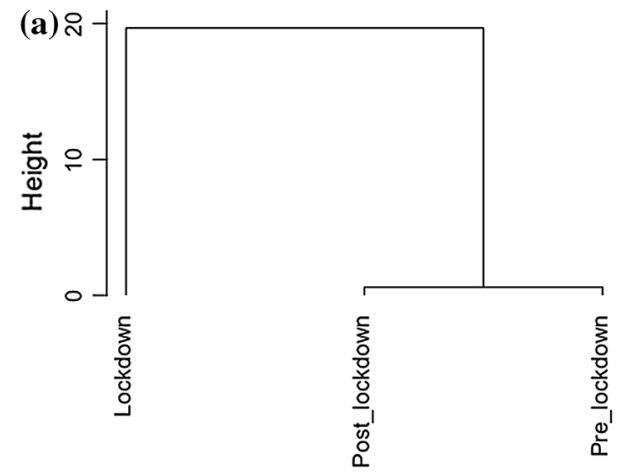




\section{Multiple regression analysis for daily confirmed/ infection and death cases of COVID-19 against the weather variables}

During pre-lockdown phase, PR has shown a significant positive contribution in spreading the daily cases while $\mathrm{RH}$ and PR has shown similar contribution in increasing daily death cases. Additionally, WS showed significant negative impact on daily infection cases. During lockdown, MT, PR, WS and RH has a significant positive impact on daily infection and death cases whereas DP has negative impact on the daily infection and death cases. During post-lockdown, DP has a positive impact on the daily infection and death cases whereas MT has positive impact only on daily death cases. Overall, it has been observed that MT, PR and RH have a consistent positive association with daily confirmed/death cases thoughout the three lockdown phases (Table S3).

\section{Lag effects of weather variables on daily confirmed/ infection and death cases of COVID-19}

The exposure-response analysis exhibiting the relative risks of COVID-19 confirmed cases and deaths in association with weather variables are shown in Figs. 3, 4, 5 and S1 - S10. Moderate infection risk was associated with MT in between 0 and 1-day lag period but no such risk was observed for its mortality till 8-day lag (Fig. 3a,b). Maximum temperature of more than $20^{\circ} \mathrm{C}$ have the higher risk of infection at 0 - and 1-day lag and less than $0{ }^{\circ} \mathrm{C}$ have the higher risk of infection from 0 to 7-day lag (Fig. S1). Similar results were also observed for mortality risk due to MT (Fig. S2). Average RH had no such risk exposure associated with daily confirmed cases and deaths for COVID19 (Fig. 3c,d), but each lag day exposure showed a mild risk of infection due to RH on 0 - to 5-day lag period. But from 7-day lag the risk of daily infection increased due to increasing RH at 10-40\% (Fig. S3). Risk of mortality due to RH increased its peak from 3-day lag at 10-40\%
Fig. 3 3D plot of maximum temperature $(\mathbf{a}, \mathbf{b})$ and relative humidity (c, d) at 10-days lag period and its corresponding relative risk (RR) generated from GAM poisson distribution model for COVID-19 daily confirmed (a, c) and death cases (b, d) till 1st November, 2020 Asia
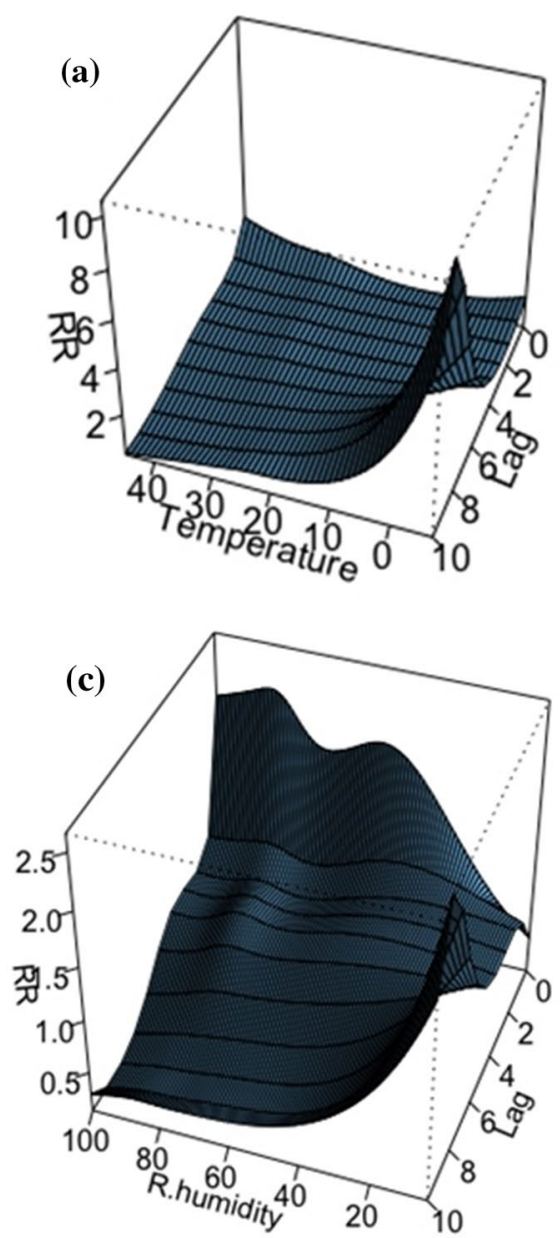
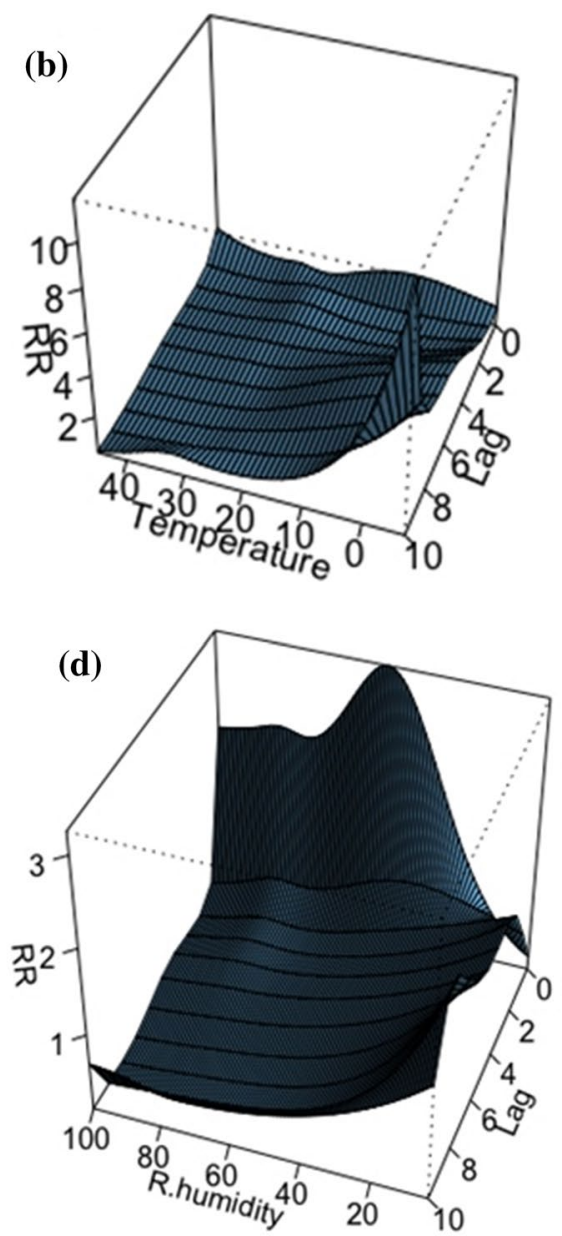
Fig. 4 3D plot of wind speed $(\mathbf{a}, \mathbf{b})$ and precipitation $(\mathbf{c}, \mathbf{d})$ at 10-days lag period and its corresponding relative risk (RR) generated from GAM poisson distribution model for COVID-19 daily confirmed (a, c) and death cases $(\mathbf{b}, \mathbf{d})$ till $1 \mathrm{st}$ November, 2020 Asia
Fig. 5 3D plot of dew point at 10-days lag period and its corresponding relative risk (RR) generated from GAM poisson distribution model for COVID-19 daily confirmed (a) and death cases (b) till 1st November, 2020 in Asia
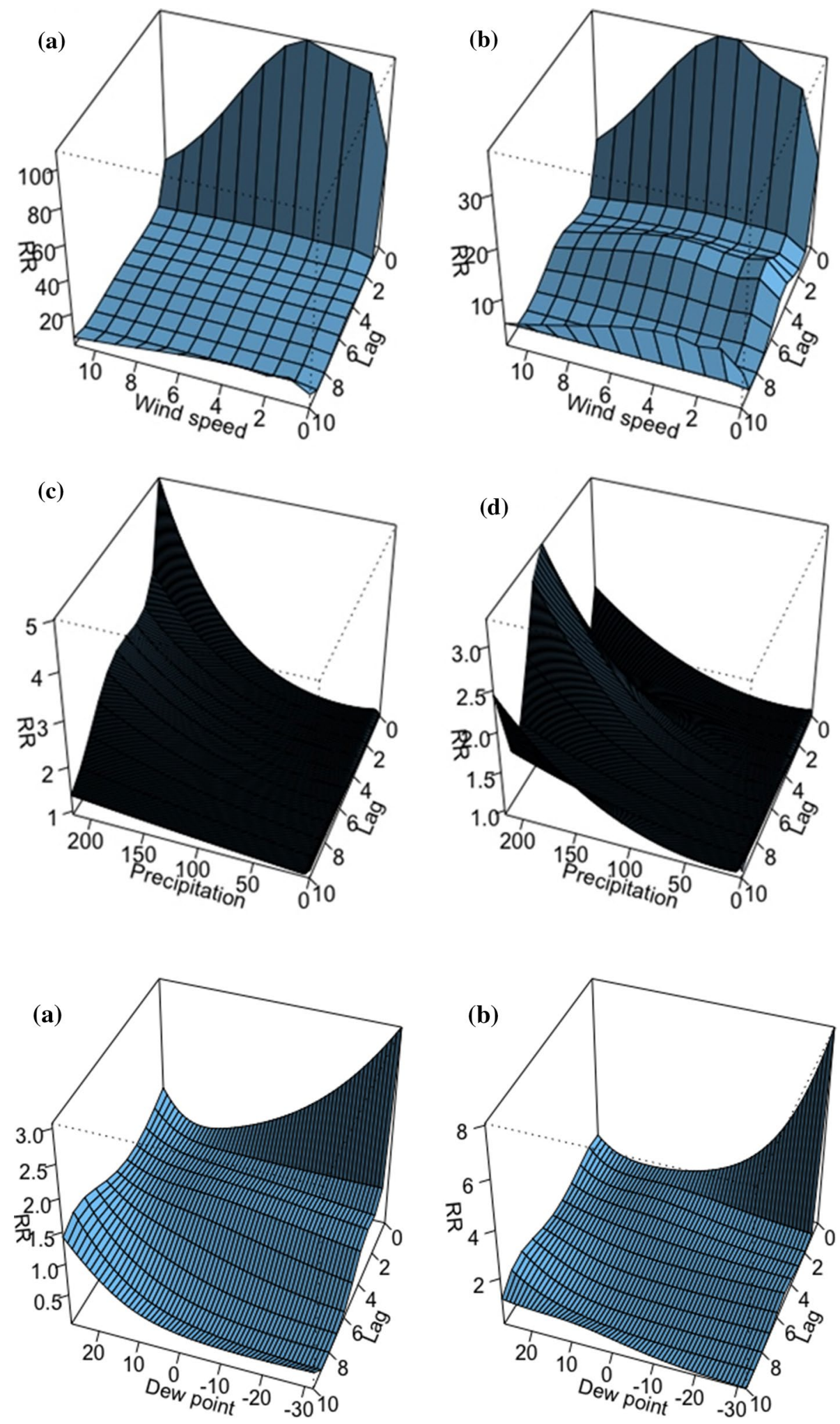
RH (Fig. S4). No infection risk was associated with WS overall but the mortality risk was observed at 8-day lag period (Fig. 4a,b). Each lag day exposure revealed minimal infection and mortality risk at 2-6 $\mathrm{m} \mathrm{s}^{-1}$ and $4-6 \mathrm{~m} \mathrm{~s}^{-1}$ of WS at 0-day lag only (Figs. S5 and S6). No infection risk and mortality risk were observed due to $\mathrm{PR}$ in lag periods (Fig. 4c,d). Lag specific exposure also revealed no significant risk associated with daily confirmed and death cases due to PR (Figs. S7 and S8). Infection risk associated with DP was observed from 4-day lag period and mortality risk was observed from 6-day lag period (Fig. 5). Each lag day effect has shown rise in infection risk from $20{ }^{\circ} \mathrm{C}$ DP at 5- and 7-day lag period (Fig. S9). The mortality risk was observed at $>0{ }^{\circ} \mathrm{C}$ DP from 1-day lag period (Fig. S10).

\section{Discussion}

Many respiratory-borne infectious diseases are correlated with temperature as shown in previous studies, where increase in the temperature results in decrease in spread of SARS-CoV, MERS-CoV and influenza virus (Chan et al. 2011; Jaakkola et al. 2014; Van Doremalen et al. 2013). Our study demonstrated positive association between confirmed and death cases with MT during lockdown. Similar results were found in Norway, Singapore, Indonesia and Brazil (Auler et al. 2020; Menebo 2020; Pani et al. 2020; Tosepu et al. 2020). Contrary to our results, some workers showed negative associations between temperature and COVID-19 cases (Lin et al. 2020; Sahin 2020; Wu et al. 2020). Association of air temperature with COVID-19 cases may vary in temperate and tropical regions where altitudinal gradient can be one of the factors for such variations. China, being the source of SARS-CoV-2 showed varied association (either positive or negative) or no association between temperature and COVID-19 cases (Liu et al. 2020; Ma et al. 2020; Qi et al. 2020; Xie and Zhu 2020; Yao et al. 2020).

A handful of studies had shown that $\mathrm{RH}$ is also an important variable apart from temperature that shows strong association with COVID-19 cases (Gupta et al. 2020; Sahin 2020; Wu et al. 2020). Here, in pre-lockdown and lockdown phase RH along with PR showed strong positive association with daily confimed and death cases. These findings provide preliminary evidence that RH and PR may increase the COVID-19 pandemic. Apart from these, WS has also shown strong negative association with either daily infections or deaths in both pre-lockdown and lockdown phase. Therefore, these weather variables should also be paid attention when estimating the cause of daily infection and death cases. In most of the cases, the risk of infection and mortality was observed in between 0 and 4-day lag periods for all weather variables, indicating the initial four days after the infection as the critical for higher risk and the cause of mortality.

Both pre-lockdown and post-lockdown have established almost similar rate in the spread of daily infection and death cases which might be due to the relaxation in social distancing in both the lockdown phases. All the three lockdown phases took place in different seasons of the year. This shows that weather variables can act as a significant response for daily confirmed and death cases in three different lockdown phases. This signifies additional support to demonstrate the role of different weather variables and their combined effect in triggering the transmission and death of COVID-19. As compared to the pre-lockdown and post-lockdown period, lockdown has evidenced more significant association between weather variables and the daily confirmed and death cases which might be due to strict social distancing revealing the approximate role of weather variables in COVID-19 cases. Due to limited movement in lockdown period, the role of weather variables in COVID-19 cases has been clearly portrayed.

It has been observed that India has the highest rate of COVID-19 cases till November 1, 2020. This country is one of the most densely populated areas in the world. Therefore, very high population dense areas might face limitations in health care facilities, serving as one of the major factors in transmission of COVID-19 cases. Also, India has diverse weather conditions in different regions which also might trigger the spread of daily cases. On the other hand, moderate to low population dense countries show lesser daily cases which might be due to well managed restrictions and social distancing with better health care facility. Similar results were observed studies from different parts of the world (Hamidi et al. 2020; Roy and Khalse 2020; Wu et al. 2020). It has been noticed that all weather variables (selected in this study) help in triggering the infection and mortality risk. This is quite clear that instead of one weather variable, the risk of COVID-19 is highly associated with the interplay of many weather variables in different seasons and their overall combined effects. This might be one of the main reasons of variations in results between the association of COVID-19 with different weather variables in different regions of the world (Auler et al. 2020; Gupta et al. 2020; Lin et al. 2020; Menebo 2020; Pani et al. 2020; Sahin 2020; Tosepu et al. 2020; Wu et al. 2020) and during different time of the year.

Overall, it has been observed that most of the respiratory-borne infectious diseases were significantly associated with changing climatic conditions like extreme temperature, drought, floods and hurricanes (see Mirsaeidi et al. 2016). Rate of change in climate alter the epidemiology of many climate-sensitive pathogens. Many studies conducted this year show strong association of SARS-CoV-2 with weather variables (Auler et al. 2020; Gupta et al. 2020; Lin et al. 2020; Menebo 2020; Pani et al. 2020; Sahin 2020; Tosepu 
et al. 2020; Wu et al. 2020). Therefore, the novel corona virus and its overall behaviour with changing weather variables show dynamic nature in transmission and lethality.

Conclusively, our work clearly indicated that combination of different environmental variables and their combined effects pose risk of infection and death, considering large set of data from Asia. For example, even $1^{\circ} \mathrm{C}$ increase in MT can pose risk for COVID-19 pandemic. Higher risk of infection due to meteorological factors is more intense in 0-4day lag period. India is unique in weather conditions with higher population density which thereby has higher infection and mortality risks as compared to the rest of the world. Significant association between the COVID-19 cases with weather variables were more defined during the lockdown when the weather conditions were changing. Therefore, it is well established that weather has a major role to play in both transmission and death cases in Asia. Along with the median age, patient's medical history and overall significance of medical facilities, factors like regional climatic conditions with daily micro-weather variables should be taken care of while dealing with the COVID-19 patients. It is important to formulate public health policies, societal awareness and preparedness for future disasters. Preparedness against the respiratory infections must include measures to check the determintal effects of micro-environmental changes and overall climatic changes so as to prevent or mitigate the spread of such infections. Other countries (apart from Asian countries) and mutants of SARS-CoV-2 have not been considered in the present study. However, these should be considered in future for better understanding of the effect of weather variables on the spread of COVID-19 cases.

Supplementary Information The online version contains supplementary material available at https://doi.org/10.1007/s42398-021-00176-8.

Acknowledgements The first author thanks the Department of Science and Technology (DST), New Delhi, India for financial support through INSPIRE Fellowship (INSPIRE Fellowship Code: IF130185).

Author contributions PP conceived the idea; AD curated the data and analysed. AD has prepared the first draft of the manuscript, PP corrected and revised the manuscript. Both the authors finalised the manuscript to be published.

\section{Declarations}

Conflict of interests On behalf of all authors, the corresponding author states that there is no conflict of interest.

Ethical statement No experiment has been done on human and animals.

Consent for publication Both the authors hereby give consent for publication.

\section{References}

Almeida SP, Casimiro E, Calheiros J (2010) Effects of apparent temperature on daily mortality in Lisbon and Oporto, Portugal. Environ Health 9:1-7. https://doi.org/10.1186/1476-069X-9-12

Auler AC, Cássaro FA, da Silva VO, Pires LF (2020) Evidence that high temperatures and intermediate relative humidity might favor the spread of COVID-19 in tropical climate: a case study for the most affected Brazilian cities. Sci Total Environ. https://doi.org/ 10.1016/j.scitotenv.2020.139090

Bashir MF, Ma B, Komal B, Bashir MA, Tan D, Bashir M (2020) Correlation between climate indicators and COVID-19 pandemic in New York, USA. Sci Total Environ. https://doi.org/10.1016/j. scitotenv.2020.138835

Bera B, Bhattacharjee S, Shit PK, Sengupta N, Saha S (2020) Significant impacts of COVID-19 lockdown on urban air pollution in Kolkata (India) and amelioration of environmental health. Environ Dev Sustain. https://doi.org/10.1007/s10668-020-00898-5

Chan KH, Peiris JM, Lam SY, Poon LL, Yuen KY, Seto WH (2011) The effects of temperature and relative humidity on the viability of the SARS coronavirus. Adv Virol. https://doi.org/10.1155/2011/ 734690

Cheng Y, Kan H (2012) Effect of the interaction between outdoor air pollution and extreme temperature on daily mortality in Shanghai. China J Epidemiol. https://doi.org/10.2188/jea.JE20110049

Djennad A, Iacono GL, Sarran C, Fleming LE, Kessel A, Haines A, Nichols GL (2018) A comparison of weather variables linked to infectious disease patterns using laboratory addresses and patient residence addresses. BMC Infect Dis 18:198. https://doi.org/10. $1186 /$ s $12879-018-3106-9$

Gasparrini A (2011) Distributed lag linear and non-linear models in R: the package dlnm. J Stat Softw 43:1

Gorbalenya AE, Baker SC, Baric R, Groot RJ, Drosten C, Gulyaeva AA, Haagmans BL, Lauber C, Leontovich AM, Neuman BW, Penzar D (2020) Severe acute respiratory syndrome-related coronavirus: The species and its viruses-a statement of the Coronavirus Study Group. bioRxiv. https://doi.org/10.1101/2020.02.07.937862 (published online Feb 11 (preprint))

Gupta A, Banerjee S, Das S (2020) Significance of geographical factors to the COVID-19 outbreak in India. Model Earth Syst Environ 6:2645-2653. https://doi.org/10.1007/s40808-020-00838-2

Hamidi S, Sabouri S, Ewing R (2020) Does density aggravate the COVID-19 pandemic? Early findings and lessons for planners. J Am Plann Assoc 86:495-509. https://doi.org/10.1080/01944363. 2020.1777891

Islam MR, Hoque MN, Rahman MS, Alam AR, Akther M, Puspo JA, Akter S, Sultana M, Crandall KA, Hossain MA (2020) Genomewide analysis of SARS-CoV-2 virus strains circulating worldwide implicates heterogeneity. Sci Rep 10:1-9. https://doi.org/10.1038/ s41598-020-70812-6

Jaakkola K, Saukkoriipi A, Jokelainen J, Juvonen R, Kauppila J, Vainio O, Ziegler T, Rönkkö E, Jaakkola JJ, Ikäheimo TM (2014) Decline in temperature and humidity increases the occurrence of influenza in cold climate. Environ Health 13:1-8. https://doi.org/10.1186/ 1476-069X-13-22

Khairat S, Meng C, Xu Y, Edson B, Gianforcaro R (2020) Interpreting COVID-19 and virtual care trends: cohort study. JMIR Public Health Surveill 6:e18811. https://doi.org/10.2196/18811

Li R, Pei S, Chen B, Song Y, Zhang T, Yang W, Shaman J (2020) Substantial undocumented infection facilitates the rapid dissemination of novel coronavirus (SARS-CoV-2). Science 368:489-493

Lin J, Huang W, Wen M, Li D, Ma S, Hua J, Hu H, Yin S, Qian Y, Chen P, Zhang Q (2020) Containing the spread of coronavirus disease 2019 (COVID-19): meteorological factors and control strategies. 
Sci Total Environ 744:140935. https://doi.org/10.1016/j.scitotenv. 2020.140935

Liu J, Zhou J, Yao J, Zhang X, Li L, Xu X, He X, Wang B, Fu S, Niu T, Yan J (2020) Impact of meteorological factors on the COVID19 transmission: a multi-city study in China. Sci Total Environ. https://doi.org/10.1016/j.scitotenv.2020.138513

Ma Y, Zhao Y, Liu J, He X, Wang B, Fu S, Yan J, Niu J, Zhou J, Luo B (2020) Effects of temperature variation and humidity on the death of COVID-19 in Wuhan. China Sci Total Environ. https://doi.org/ 10.1016/j.scitotenv.2020.138226

Mahato S, Pal S, Ghosh KG (2020) Effect of lockdown amid COVID19 pandemic on air quality of the megacity Delhi, India. Sci Total Environ. https://doi.org/10.1016/j.scitotenv.2020.139086

Maurya AP, Chikhale RV, Pandey P (2020) Transmission of SARSCoV-2 in South Asian countries: molecular evolutionary model based phylogenetic and mutation analysis. Environ Sustain. https://doi.org/10.1007/s42398-020-00123-z (accepted and published online)

Menebo MM (2020) Temperature and precipitation associate with Covid-19 new daily cases: a correlation study between weather and Covid-19 pandemic in Oslo. Norway Sci Total Environ. https://doi.org/10.1016/j.scitotenv.2020.139659

Mirsaeidi M, Motahari H, Taghizadeh Khamesi M, Sharifi A, Campos M, Schraufnagel DE (2016) Climate change and respiratory infections. Ann Am Thorac Soc 13:1223-1230. https://doi.org/10. 1513/AnnalsATS.201511-729PS

Pani SK, Lin NH, RavindraBabu S (2020) Association of COVID-19 pandemic with meteorological parameters over Singapore. Sci Total Environ. https://doi.org/10.1016/j.scitotenv.2020.140112

Prata DN, Rodrigues W, Bermejo PH (2020) Temperature significantly changes COVID-19 transmission in (sub) tropical cities of Brazil. Sci Total Environ. https://doi.org/10.1016/j.scitotenv.2020.138862

Qi H, Xiao S, Shi R, Ward MP, Chen Y, Tu W, Su Q, Wang W, Wang $X$, Zhang Z (2020) COVID-19 transmission in Mainland China is associated with temperature and humidity: a time-series analysis. Sci Total Environ. https://doi.org/10.1016/j.scitotenv.2020.138778

R Core Team (2020) R: A language and environment for statistical computing. R Foundation for Statistical Computing, Vienna, Austria. https://www.R-project.org/

Roy S, Khalse M (2020) Epidemiological determinants of COVID19-related patient outcomes in different countries and plan of action: a retrospective analysis. Cureus. https://doi.org/10.7759/ cureus. 8440

Şahin M (2020) Impact of weather on COVID-19 pandemic in Turkey. Sci Total Environ. https://doi.org/10.1016/j.scitotenv.2020.138810
Tan J, Mu L, Huang J, Yu S, Chen B, Yin J (2005) An initial investigation of the association between the SARS outbreak and weather: with the view of the environmental temperature and its variation. J Epidemiol Community Health 59:186-192. https://doi.org/10. 1136/jech.2004.020180

Tosepu R, Gunawan J, Effendy DS, Lestari H, Bahar H, Asfian P (2020) Correlation between weather and Covid-19 pandemic in Jakarta. Indonesia Sci Total Environ. https://doi.org/10.1016/j. scitotenv.2020.138436

Van Doremalen N, Bushmaker T, Munster VJ (2013) Stability of Middle East respiratory syndrome coronavirus (MERS-CoV) under different environmental conditions. Euro Surveill 18: 20590. http://www.eurosurveillance.org/ViewArticle.aspx?ArticleId= 20590. https://doi.org/10.2807/1560-7917.es2013.18.38.20590

Verity R, Okell LC, Dorigatti I, Winskill P, Whittaker C, Imai N, Cuomo-Dannenburg G, Thompson H, Walker PG, Fu H, Dighe A (2020) Estimates of the severity of coronavirus disease 2019: a model-based analysis. Lancet Infect Dis. https://doi.org/10.1016/ S1473-3099(20)30243-7

WHO (2020) World Health Organisation Characterizes COVID-19 as a Pandemic. 2020(3)

Wu Y, Jing W, Liu J, Ma Q, Yuan J, Wang Y, Du M, Liu M (2020) Effects of temperature and humidity on the daily new cases and new deaths of COVID-19 in 166 countries. Sci Total Environ. https://doi.org/10.1016/j.scitotenv.2020.139051

Xie J, Zhu Y (2020) Association between ambient temperature and COVID-19 infection in 122 cities from China. Sci Total Environ 724:138201. https://doi.org/10.1016/j.scitotenv.2020.138201

Yao Y, Pan J, Liu Z, Meng X, Wang W, Kan H, Wang W (2020) No association of COVID-19 transmission with temperature or UV radiation in Chinese cities. Eur Respir J. https://doi.org/10.1183/ 13993003.00517-2020

Zeng Q, Li G, Cui Y, Jiang G, Pan X (2016) Estimating temperaturemortality exposure-response relationships and optimum ambient temperature at the multi-city level of China. Int J Environ Res Public Health 13:279. https://doi.org/10.3390/ijerph13030279

Publisher's Note Springer Nature remains neutral with regard to jurisdictional claims in published maps and institutional affiliations. 